Vierteljahrsschrift für Geschichte der Medizin und der Naturwissenschaften Revue trimestrielle d'histoire de la médecine
GESNERUS

Jahrgang/Vol. 291972

Heft/Fasc. 1/2

\title{
Medicorum commercium Austro-Helveticum
}

\author{
Von Erna Lesky
}

Seit eh und je gelten die Wiener Sängerknaben als Botschafter spezifisch österreichischer Kultur. Zu Motetten, die sie bereits am Hofe Maximilans I. sangen, hat ein Schweizer Mediziner den Text verfaßt. Mehr noch: Dieser Schweizer stand zu ihnen in einem ganz besonderen Vertrauensverhältnis. Als seinen Schülern hat er den adolescentibus cantoribus Viennae 1515 seine Ausgabe des 7. Buches der Naturalis Historia des Plinius gewidmet ${ }^{1}$. Joachim von Watt (1484-1551) hat als Vadianus den Humanismus Wiener Stils in einer solchen Weise rezipiert und praktiziert, daß sich in ihm Motettentexte und Pliniusedition, Musik, Dichtung, Naturwissenschaft und Medizin zu einer Lebenseinheit ohnegleichen zusammenschlossen.

Man kann über Vadian nicht sprechen, ohne sich den ausgezeichneten Darstellungen Werner Näfs ${ }^{2}$ und Bernhard Milts ${ }^{3}$ verpflichtet zu fühlen. Nur das Wichtigste über seine Wiener Zeit kann hier hervorgehoben werden. Vadian hat im Studienjahr 1501/1502 das studium Viennense bezogen, das ihm zur geistigen Heimat werden sollte. Mit dem Datum ist eine Zeit der Wende für die Wiener Universität bezeichnet ${ }^{4}$. Sehr unsanft hatte Maximilian I. die Artistenfakultät aus ihrer scholastischen Erstarrung erweckt, indem er mit der Berufung des Humanisten Konrad Celtis (1459 bis

1 Werner NäF, Vadian und seine Stadt St.Gallen, 1.Band: bis 1518, Humanist in Wien, St. Gallen 1944, S. 208.

2 Zit. Anm. 1.

3 Bernhard Mrtt, Vadian als Arzt, Vadian-Studien, Untersuchungen und Texte, herausgegeben von WerNer NÄF, Band 6, St. Gallen 1959.

4 Alphons Lhotsky, Die Wiener Artistenfakultät 1365-1497, Sitzb. Österr. Ak. Wiss., phil.-hist. Kl. 247, 2.Abh., Wien 1965, S. $189 \mathrm{ff}$. 
1508) ${ }^{5}$ nach Wien einen weithin wirkenden Akt geistiger Neuorientierung setzte. Was Celtis nach Wien brachte, war nicht nur die literarische Form des klassischen Humanismus italienischer Prägung, sondern ein humanistisches Bildungsideal, in dem auch die realen Zweige der Wissenschaften ihren Platz hatten. So ist es kein Zufall, daß Celtis' Schüler, Cuspinian (1473-1529) ${ }^{6}$ und Collimitius (Georg Tannstetter) ${ }^{7}$, Medizin studierten und sich ärztlich betätigten. Und es hat seine Bedeutung, daß Vadian ebenso Schüler der beiden Genannten wie der ihres Lehrers gewesen ist. So sind in der Entwicklung, die Vadian in Wien genommen hat, zwei Richtungen erkennbar, die jedoch im Denken der Zeit durchaus als eine Einheit empfunden wurden. Auf der einen Seite steht der poeta laureatus, der am 12. März 1514 vom Kaiser selbst, von Maximilian I., in glanzvoller Versammlung zu Linz zum Dichter gekrönt wurde. Schon im nächsten Jahr folgte diesem Ereignis ein nicht weniger ehrenvolles: der Kaiser lud im Juli 1515 den König von Ungarn und Böhmen, jenen von Polen und eine Reihe weltlicher und geistlicher Fürsten nach Wien, um jene Familienverbindung mit dem Hause der Jagellonen zu feiern, die die österreichisch-ungarische Monarchie begründen sollte. An diesem Schicksalstag habsburgischer Politik war der Schweizer Joachim von Watt als Sprecher der Universität auserwählt, die laudatio auf den Kaiser und auf den Polenkönig zu halten.

Alles das, Vadians rhetorische und poetische Produktionen, bewegten sich ganz im Stil des klassisch-literarischen Humanismus, der sein höchstes Ziel darin erkannte, möglichst getreu die großen Vorbilder der Antike zu imitieren. Für Vadian als Editor und Kommentator war es jedoch entscheidend, daß der Humanismus, den er in Wien kennenlernte, starke naturalistische Züge enthielt. Sie hat er aufgegriffen und mit dem neuen, objektbezogenen Wirklichkeitssinn seiner eigenen Zeit erfüllt, als er 1510 das reizende Werk De cultura horti des ehemaligen sanktgallischen Klosterschülers Walahfrid Strabo in einer Wiener Druckerei herausbrachte ${ }^{8}$. Und erst recht hat er sich von Traditionalismus und Autoritätsgläubigkeit ge-

${ }^{5}$ Lewis W. Sprtz, Conrad Celtis, The German Arch Humanist, Cambridge 1957; ND в 3, S. $181 \mathrm{ff}$.

${ }^{6}$ Hans Ankwicz-Kleehofen, Der Wiener Humanist Johannes Cuspinian, Graz/Köln 1959; ND B 3, S. $450 \mathrm{ff}$.

7 W.NÄF 1, S. 169, 177 ff.; N D B 3, S. 322 f.

8 Vom Gartenbau, erstmals veröffentlicht von JoAchim von WATt (Vadianus), herausgegeben, übersetzt und eingeleitet von W.NÄF und M. Gabathuler, St. Gallen 19421 $1957^{2}$. 
löst und ist, wie das Bernhard Milt ${ }^{9}$ so überzeugend ausführte, zum Vertreter des neuen Wirklichkeits- und Wahrheitsprinzips in der Naturwissenschaft der Renaissance geworden, als er die Schrift des antiken Geographen Pomponius Mela 1518 bzw. 1522 edierte und kommentierte. Dieser Zug zum Realen war es denn auch, der Vadian sich nicht mit dem Studium der philosophisch-philologischen Fächer an der Artistenfakultät begnügen ließ, sondern der den bereits dozierenden trieb, sich 1512 auch noch bei den Medizinern einschreiben zu lassen. Als Vertreter der Mediziner hat denn Vadian 1516 die höchste akademische Würde erreicht, die eine Universität vergeben kann: das Rektorat. Wie sich der spätere St.Galler Stadtarzt während seines 17 jährigen Wiener Aufenthaltes stets als Schweizer gefühlt hat, so tat er es erst recht als Wiener Rektor, so daß ein Freund voll Stolz auf Vadians Schweizertum und zugleich auf seine hohe Würde bemerkte ${ }^{10}$ : ... quo nullus Helvetiorum vix unquam decoratus est.

Der in seinem Schweizertum gegründeten Stetigkeit im Schaffen und in der Entwicklung Vadians steht der heimatlos unstete Landfahrer Paracelsus gegenüber, der in Einsiedeln geboren, in Kärnten aufgewachsen und in Salzburg gestorben ist. Obwohl er in seinem Wanderleben die österreichischen Länder oftmals mit der Schweiz vertauschte und beide Länder ihn gerne als den ihren in Anspruch nehmen, ist er von der Unrast seines Wesens getrieben, in Wahrheit ein Heimatloser geblieben. So kann denn auch sein Schaffen nicht eigentlich als eine Klammer zwischen den beiden Ländern bezeichnet werden, deren geistige Kommunikation auf den Gebieten der Medizin und der Naturwissenschaften uns hier beschäftigt.

Dabei ergibt sich von selbst, daß die großen Epochen der Wissenschaftsgeschichte mit ihren führenden Männern und ihrer jeweils zeittypischen Problematik in den Vordergrund treten. Das gilt ebenso für die Medizin der Renaissance, die für uns Vadian repräsentierte, wie in besonderer Weise für die Medizin der Aufklärung. Von den Beziehungen des Schweizer Boerhaave-Schülers Albrecht von Haller (1708-1777) zu den Wiener Boerhaave-Schülern Gerard van Swieten (1700-1772) und Anton de Haen (1704 bis 1776) ist so viel zu sagen, daß ich ihnen 1958 und 1959 zwei gesonderte Untersuchungen ${ }^{11}$ im Gesnerus widmen mußte. Ohne auf einzelnes einzu-

9 B.Milt, a.a. O., S. 23 ff.; vgl. auch W.NÄF, a.a.O. 1, S. 274 ff.

10 W.NÄF, a.a.O. 1, S. 156, Anm. 2.

11 ErNa LeSKy, Albrecht von Haller, Gerard van Swieten und Boerhaavens Erbe, Gesnerus 15 (1958) 120-140; dieselbe, Albrecht von Haller und Anton de Haen im Streit um die Lehre von der Sensibilität, Gesnerus 16 (1959) 16-46. 
gehen, sei hier nur so viel hervorgehoben: Im Verhältnis de Haens zu Haller ging es um einen für die Wissenschaftsgeschichte des 18. Jahrhunderts signifikanten Problemkomplex, die Irritabilitäts- und Sensibilitätslehre und ihre klinische Interpretation. Die Differenzen, die sich hier zwischen dem Kliniker de Haen und dem Theoretiker Haller auftaten, führten zu einem richtigen bellum literarium, wie es für die Zeit so charakteristisch ist.

Ein solches hat zwar van Swieten gegen Haller nicht geführt, wenngleich der Gegensatz, in dem er zu dem großen Schweizer stand, um nichts geringer war. Hier standen sich zwei Boerhaave-Schüler gegenüber, von denen es für den einen, van Swieten, schlechtweg Gesetz der Pietät war, das wissenschaftliche Erbe des Meisters bis zum letzten Buchstaben getreu zu bewahren und zu verteidigen, während Hallers Forschertemperament ihn dazu trieb, sich kritisch von Boerhaavens Lehre zu distanzieren und sie im Zuge der fortschreitenden Erkenntnis zu verändern.

Dieser Gegensatz im wissenschaftlichen Verhalten der beiden Männer spricht sich sehr signifikant in ihrem Verhältnis zu Auenbrugger ${ }^{12}$ und seiner Erfindung aus. Van Swieten hat in seinem Konservatismus die säkulare Leistung seines eigenen Schülers, die Auenbruggersche Perkussion, in ihrer Bedeutung nicht zu erkennen vermocht und sie Zeit seines Lebens ignoriert. Haller dagegen hat den Beginn moderner Diagnostik, das Inventum novum des Jahres 1761, bereits 1762 als «eine völlig neue Erfindung» den Lesern der Göttinger Gelehrten Anzeigen mit lobenden Worten vorgestellt ${ }^{13}$.

Es ist sehr wenig bekannt, daß sich in der Folge eine Freundschaft zwischen Auenbrugger und Haller entwickelte. Einige Briefe sind uns noch erhalten ${ }^{14}$, so derjenige, mit dem Auenbrugger das Haller gewidmete Exemplar seiner 1776 erschienenen psychiatrischen Schrift Experimentum nascens $^{15}$ begleitete. Wenn in diesem Brief Auenbrugger auf seine Entlassung aus dem Spanischen Spital anspielt und einfließen läßt, daß der Grund hiefür nicht auf seiner Seite lag und das Wort sed mit einigen Punkten

12 Vgl. Erna Lesky, Leopold Auenbrugger - Schüler van Swietens, Dtsch. med. Wschr. 84 (1959) 1017-1022.

13 Vgl. B. Noltenius, Zur Geschichte der Perkussion von ihrer Bekanntgabe durch Auenbrugger (1761) bis zur Wiederbelebung durch Corvisart (1808), Arch. Gesch. Med. 1 (1908).

14 In Faksimile Reproduktion bei Max Neuburger, Leopold Auenbruggers Inventum novum, Wien/Leipzig 1922.

15 Dazu vgl. Erna Lesky, Auenbruggers Kampferkur und die Krampfbehandlung der Psychosen, Wiener klin. Wschr. 71 (1959) 289-293. 
beziehungsvoll hinzufügt, so setzt diese Anspielung eine große Vertrautheit bei Haller mit den persönlichen Verhältnissen Auenbruggers voraus. Denselben Eindruck einer über die Berufsbeziehungen hinausgehenden Freundschaft zwischen den beiden Männern gewinnt man aus dem Kondolenzbrief, den Auenbrugger nach dem Tode Hallers 1778 an dessen Sohn schrieb. Wenn er dort klagt, mihi vero verus Aestimator aequi bonique Fautor, et irreparabilis amicus ereptus est, so ist in diesem von sachlicher Wertung zu ganz persönlichem Gefühl sich steigernden Trikolon die Stellung des säkularen Vertreters der theoretischen Medizin zum Pionier klinischer Diagnostik in eindrucksvoller Prägnanz bezeichnet.

Ein Trifolium sehr verschieden gearteter Schweizer hat um die Wende vom 18. zum 19. Jahrhundert in Wien auf verschiedenen medizinischen Feldern eine bemerkenswerte Wirkung entfaltet. Da ist zunächst der ebenso lebenslustige wie lebenskluge Genfer Patriziersohn Jean de Carro (1770 bis 1857 ${ }^{16}$. Eigentlich wollte er sich 1796, nachdem er sein Doktordiplom in Edinburgh erworben hatte, nur an den Kliniken des Allgemeinen Krankenhauses etwas umsehen, bis sich eben die jakobinistischen Horden in Genf ausgetobt hätten. Dann aber ist ihm als dem geborenen Diplomatenarzt das Leben in der Wiener High Society so lieb und auch so lukrativ geworden, daß er - anders als der St. Galler Bürgersohn - nicht mehr an Heimkehr dachte. Der Genfer homme du monde ist 1857 in Karlsbad als Kurarzt gestorben. Dazwischen liegt ein Leben voll Geschäftigkeit, die in ihrer weiten Streuung keineswegs der für die Zeit charakteristischen philanthropischen Züge entbehrte. Wir glauben, Jean de Carro nicht Unrecht zu tun, wenn wir ihn als einen philanthropischen Manager bezeichnen, dem es nichts ausmachte, ob er die neuesten Pariser Schwefelräucherungen in Wien propagierte oder eine neue Reis- oder schottische Schafart oder ob er die Karlsbader Quellen anpries. Unbestritten bleibt sein philanthropisch-propagandistisches Meisterstück: die Bekanntmachung der eben 1798 von Jenner publizierten Kuhpockenimpfung in der Türkei, in Griechenland und in Indien. Wenn die Großtat Jenners bei der Langsamkeit der damaligen Kommunikationsmittel bereits im Jahre 1800 so weit im Osten bekannt wurde,

16 Vgl. Mémoires du Chevalier Jean de Carro, Carlsbad 1855; L. Petersohn, Chevalier Jean de Carro, Med. Diss. Wien o. J.; H.E.Sigerist, Letters of Jean de Carro to Alexandre Marcet, 1794-1817, Suppl. Bull. Hist. Med. No.12, Baltimore 1950; JeAn OuIvier, A propos du Dr. Jean de Carro, Gesnerus 8 (1950) 164-168; K. Neydl und J.Miesler, Jean de Carro a Karlovy Vary, Karlsbad 1958 (in tschechischer Sprache); ERNA Lesky, Die Wiener medizinische Schule im 19. Jahrhundert, Graz/Köln 1965, S. 28 ff. 
so ist dies darauf zurückzuführen, daß de Carros flinker Verstand alle Möglichkeiten ausschöpfte, die ihm Wien als das Kontaktzentrum zwischen West und Ost damals bot.

Einen ganz anderen Typus in der Mannigfaltigkeit schweizerischen Arzttums repräsentiert der streitbare und querköpfige Ignaz Paul Vital Troxler $(1780-1866)^{17}$ aus Beromünster, der zwischen 1804 und 1815 dreimal längeren Aufenthalt in Wien nahm. Medizinhistorisch interessant ist nur der erste Aufenthalt 1804/1805. Er fällt in eine Umbruchszeit der Wiener Medizin. Brownianismus und Erregungstheorie hatten eben die traditionelle Humoralpathologie erschüttert. Da kam nun Troxler unmittelbar von Schelling und tat mit seinen naturphilosophischen Ideen ein übriges, um jene Bewegung in Wien zu inaugurieren, in der die Spekulationen der Romantischen Medizin keine geringe Verwirrung unter den Geistern stifteten.

Troxlers Freund und Kampfgenosse war Johann Malfatti (1775-1859) ${ }^{18}$, der ehemalige Frank-Assistent. Er hatte damals bereits eine Nobelpraxis in Wien ${ }^{19}$ mit sehr einflußreichen Beziehungen. So bedeutete es nicht wenig, daß dieser angesehene Arzt einer der Hauptrepräsentanten der Romantischen Medizin in Wien wurde. Da in seinem Hause, in dem auch Troxler wohnte, die in der Frank-Ära gegründete Gesellschaft der Ärzte ihre Sitzungen abhielt, erreichte Troxler mit seinen Ideen auch diesen wissenschaftlich außerordentlich interessierten Kreis. Man kann vermuten, daß ihm Troxler selbst sein 1805 in Wien erschienenes Werk Grundri $\beta$ der Theorie der Medizin vorgelegt hat, das eine völlige Reform der Heilkunde auf naturphilosophischer Basis anstrebte.

In der Tat, in einer solchen Zeit war es notwendig, zur Raison zu rufen, zur Besinnung auf die Vergänglichkeit luftiger Theorien. Ein anderer Schweizer Arzt, Heinrich Attenhofer (1783-1856) ${ }^{20}$, hat dies getan. Er ist damit 1808 - wir stellen es mit besonderer Freude fest - zum ersten Fachdozenten und Begründer der Wiener Medizingeschichte geworden.

17 Vgl. I. Belke, I. P. V.Troxler, Neue Dtsch. Forsch. Abt. Philosophie, Berlin 1935; EmIL SpIEss, I.P.V.Troxler, Bern/München 1967.

18 Über ihn vgl. E. LeSKY (zit. Anm. 16) S.101 ff.

i9 Auch Beethoven zählte zu den Patienten Malfattis. So wurde auch Troxler mit ihm bekannt und konnte ihm sogar Dolmetscherdienste leisten. Vgl. E.SpIEss, a.a. O., S. 67, 984, Anm. 16.

20 Über ihn vgl. Heinrich Buess, Heinrich Ludwig Attenhofer (1783-1856), Schweiz. med. Wschr. 86 (1956) S. 932 ff.; ferner E. LeSKy (zit. Anm. 16) S. 617 f. 
Wie H. Buess ausführte, stammte Attenhofer aus dem luzernischen Zweig der bekannten Schweizer Familie. 1805 hatte Attenhofer im Zuge seiner postpromotionellen Ausbildung wie so viele Ausländer vor und nach ihm das Wiener Allgemeine Krankenhaus aufgesucht und war 1807 an ihm sogar - bei Ausländern eine große Seltenheit - zum Sekundararzt avanciert. 1808 lag bereits eine Publikation Attenhofers über das lymphatische System vor. Der erste Abschnitt, der über die Geschichte des Problems handelt, verrät bereits den künftigen Historiographen. So kann Attenhofer in seinem Gesuch um Bewilligung medizinhistorischer Vorlesungen füglich auf dieses Werk hinweisen. Das mag sicherlich den strebsamen jungen Luzerner als künftigen Dozenten für Geschichte der Medizin bei dem allgewaltigen Protomedicus Stifft empfohlen haben. Ausschlaggebend war jedoch für diesen eine andere Überlegung. Attenhofer hatte in seinem Gesuch die Notwendigkeit der Medizingeschichte folgendermaßen begründet : «... (sie lehrt) einsehen, wie die meisten Theorien, die der Neuheit wegen so leicht Eingang fanden ... nur in einem lockenden Modegewand erscheinen. » In einer Zeit sich überstürzender und vielfach kurzlebiger Systeme mußte eine solche Besinnung auf die Tradition natürlich dem Manne besonders willkommen sein, der wie Stifft Zeit seines Lebens Traditionalist um jeden Preis gewesen ist.

In der Vielfalt, in der sich österreichische und Schweizer Medizin zu den verschiedenen Epochen begegnen, nimmt die Wiener Lehrzeit des großen schweizerischen Sozialmediziners Jakob Laurenz Sonderegger (1825 bis 1896 ${ }^{21}$ einen besonderen Platz ein. Und dies nicht allein, weil er «das großartige Schauspiel der Wiener Revolution» - gemeint ist die Oktoberrevolution des Jahres 1848 - «von Anfang bis zu Ende zuschauend mitmachte» (Sonderegger, S.188); weit bedeutsamer ist es gewesen, daß die Zeit seines Wien-Aufenthaltes (Oktober 1848 bis April 1849) mit der Blütezeit der Zweiten Wiener Medizinischen Schule zusammenfällt. Man darf also hoffen, aus den Briefen und autobiographischen Aufzeichnungen, die uns bei Sonderegger glücklicherweise in so großer Fülle zur Verfügung stehen, ein getreues Bild vom Betrieb dieser Schule und von ihren Lehrmethoden zu erhalten. Unsere erste Frage wird daher lauten: Was konnte diese Schule

${ }^{21}$ Dr.L. Sonderegger in seiner Selbstbiographie und seinen Briefen, herausgegeben von Elias Haffter, Frauenfeld 1898 (zit. im Text als Sonderegger); PAUL PopP, Der Beitrag von Jakob Laurenz Sonderegger (1825-1896) zur Sozialmedizin und Sozialpolitik, Med. Diss. Basel 1960; Heinrich Buess, J.L.Sonderegger (1825-1896) als Standespolitiker, Verh. Schweiz. Naturforsch. Ges. 1969, S. 174. 
und ihr Heim, das Allgemeine Krankenhaus, einem Schweizer Medizinstudenten von der Aufnahme- und Kritikfähigkeit eines Sonderegger bieten?

Da ist zunächst das Erlebnis der großen Zahl, der Krankheit an sich in der ganzen Mannigfaltigkeit ihrer Erscheinungsformen. Sie tritt Sonderegger bei dem Chirurgen und späteren Venerologen Sigmund täglich in 100 bis 120 Fällen entgegen, bei dem Chirurgen Dumreicher in ebenso vielen, bei dem Dermatologen Hebra sind es sogar 150 oder mehr Kranke «mit allen möglichen Hautleiden von den Epizoen an bis zu den Variolen und Morbillen in der reichsten Schattierung». Und vollends überwältigt hat Sonderegger der Betrieb an den beiden Wiener Gebärkliniken (Sonderegger, S.189): «Das Gebären geht en gros immer fort. Die erste Klinik hat jährlich 3500 Geburten; ebenso viele sind an der zweiten für die Hebammen bestimmten Anstalt. Man kann oft in 12 Stunden 18 Fälle beobachten.» Gerade aber dieser Reichtum klinischen Krankengutes war es, um dessentwillen Sonderegger Wien aufsuchte, da einen vergleichbaren ihm die damals weniger reich frequentierte Zürcher Klinik nicht bieten konnte.

Ein so anspruchsvoller Student, der sich wie Sonderegger mit großer Gewissenhaftigkeit und einer fast altklug anmutenden Umsicht für die Führung seiner zukünftigen Landpraxis vorbereitete, hätte sich mit der bloßen Quantität nie zufriedengegeben, wenn sie nicht auch mit einer bestimmten Qualität medizinischen Unterrichts verbunden gewesen wäre. Nun ruhten aber an der Universität infolge der revolutionären Wirren alle offiziellen Lehrveranstaltungen von Oktober 1848 bis Februar 1849. Durch diesen Ausnahmezustand hat Sonderegger die einmalige Gelegenheit gehabt, die Wiener Medizinische Schule gleichsam von ihrer intimen Seite her kennenzulernen, und zwar in der Form, in der ihr Lehrbetrieb brillierte und sich als höchst effizient erwies: bei den Visiten am Krankenbett, den Sektionen in der Totenkammer und in den Privatkursen der verschiedenen Professoren, Dozenten, Primarien und Assistenten. Es waren dies Kurse, in denen kleine Gruppen von 10 bis 20 Hörern täglich durch 6 bis 8 Wochen hindurch unmittelbar am Objekt, am Krankenbett oder am Leichentisch, instruiert wurden. Was wir heute im Gruppenunterricht als modernste Unterrichtsform entdeckt zu haben glauben, war im Wien der Rokitansky-SkodaSchule eine selbstverständlich und systematisch praktizierte Wirklichkeit. So konnte Sonderegger trotz aller politischen Wirrnisse des 48 er-Jahres nicht nur sein selbstvorgenommenes didaktisches Plansoll erfüllen, sondern im Allgemeinen Krankenhaus wahre Lernbacchanalien von 1/27 Uhr früh 
bis 5 Uhr nachmittags feiern und in diesem Zusammenhang sogar von «eine(r) genußreiche(n) Welt im Krankenhause» sprechen (Sonderegger, S. 192).

Was der zukünftige Landarzt am meisten brauchte, die Ausrichtung auf das Praktische, sie stand im Wiener Lehrbetrieb ganz im Vordergrund, mag es nun der «vorzügliche Demonstrator» Hebra oder der Assistent Wenzel Linhart, der spätere Würzburger Chirurg, mit seinem chirurgischen oder der «famose» Semmelweis mit seinem geburtshilflichen Operationskurs gewesen sein. Für das gute Urteil Sondereggers zeugt, daß er bei der Nennung von Semmelweis' Namen noch hinzufügt (Sonderegger, S.189): «Überhaupt macht dieser Mann von wissenschaftlicher und humaner Seite aus einen äußerst guten Eindruck.» Die Treffsicherheit und Ausgewogenheit von Sondereggers Kritik wird nur bestätigt, wenn er an dem Okulisten Rosas, den wir heute als den Hauptgegner von Semmelweis kennen ${ }^{22}$, wohl dessen operative Geschicklichkeit lobt, aber hinzufügt (Sonderegger, S. 189): «(Er ist aber) ein Plauderer und Alterthümler wie kaum Einer ..., ist ein Name und wenig mehr für die jetzige Medicin.»

Kein Zweifel: dieser junge Schweizer Student hat sehr wohl den scharfen Gegensatz gespürt, der sich eben in seiner Wiener Lehrzeit im Kampf um Semmelweis zwischen einer progressiven und einer konservativen Professorengruppe auftat, zwischen Rokitansky und Skoda einerseits als den Vertretern der neuen Faktenmedizin und Rosas und Klein, dem Chef Semmelweis', andererseits, den Männern von Gestern ${ }^{23}$.

So spürt man aus Sondereggers Aufzeichnungen deutlich, wie sich im unmittelbaren Kontakt gerade mit den wissenschaftlich konträren Lehrerpersönlichkeiten Wiens sein eigener Standort in Medizin und Wissenschaft immer klarer abhebt, wie sich in seinem Wissenschaftsbild die neue zukunftsträchtige Medizin naturwissenschaftlicher Prägung von der alten, konventionellen scharf absetzt. Das spricht sich deutlich in der Selbstcharakteristik aus, die er in seiner «Bilanz» präsentiert (Sonderegger, S. 99): «Ich gehörte zu der ersten Generation, der es beschieden war, die anatomische und physiologische Auffassung am Krankenbette zu verwerten und die physikalische Diagnostik zu handhaben, wobei man allerdings weiter kam, als bei der hergebrachten Hufeland'schen Praxis.»

22 Vgl. Erna Lesky, Ignaz Philipp Semmelweis und die Wiener medizinische Schule, Sitzb. Österr. Ak. Wiss., phil.-hist. Kl. 245, 3. Abh., Wien 1964, S.14 ff.

${ }_{23}$ Ebenda, S. 31. 
Was Sonderegger in seine Rheintaler Praxis aus Wien mitbrachte, waren eine neue Auffassung der Krankheit und neue Untersuchungsmethoden. Beide hat er unmittelbar bei ihren Schöpfern selbst, bei Rokitansky und Skoda, gelernt. So braucht man sich nicht zu wundern, daß seit den fünfziger Jahren in Balgach und Altstätten die Obduktionen zur Usance wurden, mochte es sich um Arme oder Reiche handeln. Bei jedem konnte der wißbegierige Landarzt etwas Neues lernen. Es ist Rokitanskyscher Geist, der ihn bekennen ließ (Sonderegger, S.38): «Mein liebstes Fortbildungsmittel waren die Leichenöffnungen.» Und wenn seit 1850 in St. Gallen perkutiert und auskultiert wurde, so sind es die beiden Skoda-Schüler Sonderegger und Seitz (Sonderegger, S. 54), die von Wien aus in die Ostschweiz die physikalische Diagnostik verpflanzten.

Nicht minder eindrucksvoll wird der Einfluß Skodas in Sondereggers Therapie sichtbar. Bei ihrem damaligen Stand war es freilich für einen Landarzt nicht leicht, vor seinen Patienten im Geiste Skodas offen und ehrlich zu bekennen: Wir sind nicht in der Lage zu ... Sonderegger hat mit feinem psychologischem Spürsinn, der immer den wahren Arzt von dem Gesundheitstechniker unterscheidet, einen Mittelweg gewählt (Sonderegger, S. 46): «Ich gebe Gebildeten sehr oft gar nichts, Ungebildeten etwas Milchzucker, den ich en gros kaufte, damit sie stille halten und mir nicht mit Aderlassen und Pillen den ruhigen Ablauf des Processes stören.» Hinter dieser Begründung steht ganz offensichtlich die Vorstellung von dem ungestörten Waltenlassen der Naturheilkraft, die sich in signifikanter Weise mit dem Wiener therapeutischen Skeptizismus bzw. Nihilismus verbindet ${ }^{24}$. Und wäre noch ein Zweifel über die Herkunft von Sondereggers therapeutischer Haltung, so würde ihn sein eindeutiges Bekenntnis aus der Welt schaffen (Sonderegger, S. 47): «Der beste Kompaß, zwischen Aberglauben und Unglauben hindurchzurudern, war für mich der Nihilismus von Scoda (sic!) und Hamernjik (sic!). ${ }^{25}$ Gleichwohl hat Sonderegger in seiner Praxis «trotz vielen und tiefen Unglaubens» (Sonderegger, S. 47) nicht auf Medikamente verzichtet, sondern die usuellen in bestimmten Fällen bei klarer Indikationsstellung eingesetzt.

24 Dazu vgl. Erna Lesky, Von den Ursprüngen des therapeutischen Nihilismus, Arch. Gesch. Med. 44 (1960) 1-20.

25 Bei dem Prager Schüler Skodas, Josef Hamernik (1810-1887), handelt es sich wie bei Josef Dietl um einen radikalen Vertreter der genannten Wiener therapeutischen Richtung. Vgl. E. LesKy (zit. Anm. 16) S. $214 \mathrm{f}$. 
Andernorts ${ }^{26}$ konnten wir, angeregt durch eine Arbeit E. H. Ackerknechts ${ }^{27}$, zeigen, daß sich auch der Wiener therapeutische Skeptizismus bzw. Nihilismus in typischer Weise mit einem ausgesprochenen Optimismus und einem großen Vertrauen zu allen hygienisch-prophylaktischen Maßnahmen verbindet. Es ist kein Zufall, wenn wir genau dieser Verbindung auch bei Sonderegger (S. 46) begegnen : «Sehr oft drängte sich mir die Überzeugung auf, daß meine Klienten infolge schlechter Wohnung, Nahrung oder Kleidung, durch ihre Berufsbetreibung oder ihre Lebensgewohnheiten krank geworden seien, und da $\beta$ die Behandlung dort einsetzen müsse, nicht mit dem Medizinkasten. ${ }^{28}$ Auch Skoda hat den Typhus nicht mit Pillen und Aderlassen bekämpft, sondern dafür gesorgt, daß die Wiener Elendsquartiere mittels der Hochquellenleitung frisches und reines Wasser erhielten.

Wir glauben daher nicht fehlzugehen, wenn wir den Grund zu der späteren so reichen sozialhygienischen Tätigkeit Sondereggers bereits in seiner Wiener Lehrzeit, in dem hygienisch-prophylaktischen Zug des Wiener therapeutischen Skeptizismus bzw. Nihilismus gelegt finden.

Dieser prophylaktisch-hygienische Zug hat seine säkulare Ausformung in der Entdeckung des erfolgreichsten (und unglücklichsten) Skoda-Schülers, des Ignaz Philipp Semmelweis (1818-1865), gefunden. Das SemmelweisErlebnis ist es denn auch, das von allen Wiener Eindrücken auf Sonderegger die nachhaltigste Wirkung ausgeübt hat. Er hat nie vergessen, was ihm dort durch seinen geburtshilflichen Instruktor vorexerziert worden war: daß man durch eine simple hygienische Maßnahme, wie es das Händewaschen mit Seife, Chlorkalk und Nagelbürste war (Sonderegger, S. 22), Hunderte, ja Tausende von Müttern vor dem Tod durch Kindbettfieber bewahren konnte. Noch in seiner Lebensbilanz erinnert er sich mit unverminderter Frische an dieses Wiener Erlebnis des Jahres 1848: «Ich genieße nach einem halben Jahrhundert noch ungeschwächt die Freude, die ich empfunden habe, als ich die Todesfälle des Kindbettfiebers gewaltig abnehmen $\operatorname{sah} . . . »$

Ziehen wir nun den Schluß aus den hier zusammengeordneten Fakten. Wir möchten glauben, daß die Wiener Lehrzeit nicht nur dem Rheintaler

${ }^{26}$ In der Anmerkung 24 zitierte Arbeit.

27 E.H.Ackerknecht, Die Therapie der Pariser Kliniker zwischen 1795 und 1840, Gesnerus 15 (1958) 151-163.

${ }^{28}$ Vgl. eine weitere, ganz ähnliche Äußerung Sonderegger, S. 100. 
Landarzt das Rüstzeug in die Hand gab, um seine Praxis mit den fortschrittlichsten Methoden der Medizin auszuüben; es dürfte auch klargeworden sein, daß die in Wien empfangenen Impulse nicht wenig zur Entwicklung des großen schweizerischen Pioniers der Sozialmedizin beigetragen haben.

Der jetzt zu nennende Schweizer Naturforscher, Arzt und Diplomat, Johann Jakob von Tschudi (1818-1889) ${ }^{29}$, steht zwar mit der Wiener Medizin nur in sehr loser Verbindung; und doch gehört auch er in unsere Reihe des medicorum commercium Austro-Helveticum. Denn im selben Revolutionsjahr 1848, als Sonderegger seine Lehrzeit im Allgemeinen Krankenhaus absolvierte, siedelte sich der Peru- und Anden-Forscher in einem einschichtigen Bauernhof bei Edlitz an und hat dort als Landarzt viele Jahre hindurch niederösterreichische Bauern auf das gewissenhafteste und liebevollste betreut. Dort sind seine Antiguedadas Peruanas (1851), sein Werk über die Kechuasprache, sein fünfbändiger Reisebericht über Südamerika entstanden, und dort ist er schließlich nach einem im Dienste des Schweizer Volkes - Tschudi war schweizerischer Gesandter in Brasilien und später durch viele Jahre am Wiener Hof - 1889 gestorben.

Als die Schweiz im vierten Jahrzehnt des 19. Jahrhunderts begann, ihre moderne Medizin an den Universitäten aufzubauen, hat auch Österreich seinen Beitrag geleistet. Wenn auch dieser Beitrag nicht so extensiv wie der Deutschlands war, so verdienen doch zwei österreichische Gelehrte hier genannt zu werden: Sondereggers Lehrer, der Rokitansky-Schüler Josef Engel (1816-1899), hat von 1844 bis 1849 in Zürich als pathologischer Anatom gewirkt. Es will viel sagen, wenn der so kritische und so ehrliche Sonderegger ihn «als einen akademischen Lehrer von Gottes Gnaden» bezeichnet (Sonderegger, S. 310).

Der Geburtshelfer und Gynäkologe August Breisky (1832-1889) ${ }^{30}$ wurde 1867 von der medizinisch-chirurgischen Lehranstalt in Salzburg an die Universität Bern berufen. Er fand dort eine veraltete geburtshilfliche Klinik vor, an der 1862 die Sterblichkeit an Kindbettfieber noch 6,33\% betrug, 1866 sogar auf $10,81 \%$ angestiegen war. Breisky führte sofort das Semmelweissche Verfahren ein. 1868 war die Sterblichkeitsziffer auf 3,94\%

29 Paul-Emile Schazmann, Johann, Jakob von Tschudi, Forscher, Arzt, Diplomat, Zürich 1956.

$30 \mathrm{Vgl}$. Friedrich Schauta, Gedächtnisrede auf August Breisky, Prager med. Wschr. 14 (1889) 257 ff.; Ursula Zimmermann, August Breisky, Sein Leben und Wirken als Arzt und Lehrer, Med. Diss., Wien o.J.; E.Lesky (zit. Anm. 16) S. 468 f. 
gesunken. Auch der Neubau der Berner Frauenklinik geht auf Breiskys Initiative zurück und gründet sich auf seine Planung. Bevor jedoch das neue Haus bezogen werden konnte, kehrte Breisky 1874 an seine Heimatuniversität Prag zurück.

Bei unserem allzu knappen Abriß über die schweizerisch-österreichischen Beziehungen im Bereiche der Medizin ist nicht zu übersehen, daß die Chirurgen eine starke Klammer zwischen den beiden Ländern bilden. Wieder können wir aus einer Fülle nur einige für die jeweilige Epoche typische Vertreter herausgreifen, allen voran Theodor Billroth (1829-1894) ${ }^{31}$.

Er hat an beiden Universitäten, in Zürich und in Wien, die moderne Chirurgie inauguriert. Immer wieder hebt man als ihre konstituierenden Elemente die Anästhesie und Antiseptik hervor. Billroth hat ihr aber in Zürich und Wien noch ein Drittes hinzugefügt. Bisher hatten die Chirurgen meistens nur über ihre erfolgreichen Operationen berichtet. Billroth hat in den Jahresberichten über die Zürcher Klinik von 1860 bis 1868 ebenso wie in ihrer Fortsetzung, den Jahresberichten über die Wiener Klinik ab 1868, offen und ehrlich auch über seine Mißerfolge Rechenschaft abgelegt. Damit hat er unter der Devise «Wahrheit und Klarheit» von Zürich und Wien aus beispielgebend die Grundlage einer brauchbaren chirurgischen Operationsstatistik geschaffen und bewiesen, daß eine solche für den weiteren Fortschritt der Chirurgie ebenso unentbehrlich ist wie Schmerzlosigkeit und keimfreie Hände.

Als Billroth 1867 Zürich mit Wien vertauschte, da ist auch sein treuer Schüler und Assistent, der Solothurner Albert Züblin, mit ihm in die Donaustadt gezogen. Nicht nur durch ihn, sondern vor allem durch seinen Zürcher Freund, den Okulisten Friedrich Horner (1831-1886), blieb Billroth dauernd der Schweiz und der Limmatstadt verbunden, hat mitgeholfen zu raten, wenn es um die Besetzung von Lehrkanzeln ging, und sich im Grunde seines Herzens trotz allen Glanzes der Kaiserstadt oft nach Zürich zurückgesehnt. Dieser Sehnsucht hat er 1872 in einem Brief an Horner ${ }^{32}$ so impulsiv und ehrlich, wie es nun einmal seine Art war, Ausdruck gegeben: «Ich muß doch bald wieder einmal nach Zürich kommen; wenn hier Alles drüber und drunter geht und ich alle meine Nerven anspannen muß, um

31 A.Huber, Theodor Billroth in Zürich, 1860-1867, Zürich 1924; E. Lesky (zit. Anm. 16) S. $435 \mathrm{ff}$.

32 Alfred Bader, Entwicklung der Augenheilkunde im 18. und 19. Jahrhundert, Basel 1933, S. 142 . 
in solchem äußern Trübsal noch zu arbeiten, und oft Wochen lang nach einer Stunde der ,Sammlung' ringe, so denke ich wohl, ich sei doch ein rechter E ... gewesen, daß ich nicht in der Zürcher Stelle blieb.»

Gerade in der Übersicht über die Chirurgen-Beziehungen drängt sich auch noch ein anderer Aspekt auf. Es ist einfach so, daß die Schweiz und Österreich als Alpenländer bestimmte gemeinsame Probleme, auch im Bereich der Medizin, haben. Ein solch gemeinsames Problem ist das Kropfproblem bzw. die Schilddrüsenchirurgie. Nach Einführung der Antiseptik lernte man in den siebziger Jahren allmählich, die schwierigen technischen Probleme der Totalexstirpation dieses Organs zu lösen ${ }^{33}$. Seit 1879 gab es keine Hemmung mehr: fleißig rottete man in Wien, Bern und Genf Schilddrüsen aus. So ist es nur natürlich, daß man in der Schweiz und in Österreich die Folgen solcher Totalexstirpationen zuerst beobachten konnte: in Wien die Tetanie, die der Neurologe Nathan Weiß 1881 als erster beschrieb und die der Billroth-Schüler Anton von Eiselsberg (1860-1939) weiter erforschte ${ }^{34}$; in der Schweiz die kretinoiden Zustände, das Myxoedème opératoire und die Kachexia strumipriva, die von Jacques-Louis Reverdin (1832 bis 1929) ${ }^{35} 1882$ und von Theodor Kocher (1841-1927) 1883 beschrieben wurden.

In der Folge kam es zu dem die deutsche und französische Chirurgie ziemlich erregenden Prioritätsstreit zwischen dem Genfer und dem Berner Chirurgen. Wir können es uns ersparen, die Einzelheiten dieses hartnäckigen Streites hier auszubreiten. Der Basler Dissertant Sigmund Bornhauser ${ }^{36}$ hat dies 1951 in einer musterhaft dokumentierten Analyse getan. Ihr Ergebnis, daß dem Genfer Jacques-Louis Reverdin die Priorität gebühre, konnten 1970 Markwart Michler und Jost Benedum aufgrund von Briefen Reverdins und Kochers aus den Jahren 1894 und 1895 bestätigen, die sie in der Gießener Universitätsbibliothek auffanden. Es ist kein Zufall, daß

33 Vgl. Sigmund Bornhauser, Zur Geschichte der Schilddrüsen- und Kropfforschung im 19. Jahrhundert, Veröffentlichungen der Schweizerischen Gesellschaft für Geschichte der Medizin und der Naturwissenschaften, Fasz. 19, Aarau 1951, S. 54 f.; F. Merke, Geschichte und Ikonographie des endemischen Kropfes und Kretinismus, Bern/Stuttgart 1971, S. $248 \mathrm{f}$.

34 Vgl. E.LesKy (zit. Anm. 16) S. 394, 443.

35 Henri Reverdin, Jacques-Louis Reverdin, 1842-1929, Un chirurgien à l'aube d'une ère nouvelle, Veröff. Schweiz. Ges. Gesch. Med. Naturw. 25, Aarau 1971.

${ }^{36}$ Zit. Anm. 33. Dazu vgl. Markwart Michler und Jost Benedum, Die Briefe von Jacques-Louis Reverdin und Theodor Kocher an Anton v. Eiselsberg, Gesnerus 27 (1970) 169-184. 
in diesen Briefen von beiden Chirurgen Anton von Eiselsberg gleichsam als Schiedsrichter aufgerufen wird. Denn er war damals die österreichische Autorität auf dem Gebiete der Schilddrüsenforschung. 1890 war seine grundlegende Monographie Über Tetanie im Anschlu $\beta$ an Kropfoperationen erschienen, und man wußte sowohl in Genf als auch in Bern, daß er eben dabei war, seine gesamten bisherigen Forschungsergebnisse über die Erkrankungen der Schilddrüse in einer monographischen Darstellung ${ }^{37}$ niederzulegen.

Es konnte den beiden Schweizern nicht gleichgültig sein, wie in diesem wohl für lange maßgeblichen Werk ihr Prioritätsstreit behandelt werde. Es gereicht dem österreichischen Chirurgen zur Ehre, daß er in großer Objektivität und Souveränität zu einem Urteil gelangte ${ }^{38}$, das durch Bornhausers Analyse weitgehend bestätigt wurde. Mit der Noblesse, die dem österreichischen Baron eigen war, hat er dem Genfer Pionier 1927 noch eine späte Freude bereitet. Als in diesem Jahr in Bern die internationale Kropfkonferenz tagte, war es Eiselsberg, auf dessen Veranlassung sich der Kongreß in einem Grußtelegramm vor dem Entdecker des Myxoedème opératoire verneigte ${ }^{39}$.

Vor mehr als zehn Jahren versammelten sich am 8. Juli 1961 im Hörsaal der Chirurgischen Universitätsklinik des Kantonsspital Zürich eine große Schar prominenter Schweizer Chirurgen. Sie waren gekommen, um ihres Lehrers Paul Clairmont (1875-1942) ${ }^{40}$ zu gedenken, der vor zwanzig Jahten gestorben war.

Clairmont war aus Wien gekommen, hatte an der Wiener Universität 1898 seinen medizinischen Doktorgrad erlangt und seine chirurgische Ausbildung an der Klinik Eiselsberg absolviert. Man hat an der Zürcher Klinik die Billrothsche Tradition fortgesetzt, als man 1919 nach dem Abgang seines Enkelschülers ${ }^{41}$ Ferdinand Sauerbruch den Enkelschüler Clairmont aus Wien berief.

37 Sie erschien unter dem Titel Die Krankheiten der Schilddrüse, in: Billroth-Lücke: Deutsche Chirurgie, Band 38, Stuttgart 1901.

38 Abgedruckt ebenda S. 150, Anm. 1 von S. 149. Auch reproduziert bei S. BorNHAuser (zit. Anm. 33) S. 80.

39 Vgl. S. Bornhauser, S.113; Henri Reverdin (zit. Anm. 35) S.132.

40 Die aus diesem Anlaß gehaltenen Ansprachen erschienen als Sammelpublikation unter dem Titel «Zum Gedenken an Paul Clairmont, 1875-1942», Praxis, Schweizer Rundschau für Medizin 51 (1962) 14-23.

41 Sauerbruch war von 1903 bis 1905 Schüler und Assistent des Billroth-Schülers Johann von Mikulicz-Radecki in Breslau gewesen. 
Clairmont hat an der Zürcher Klinik 22 Jahre gewirkt. In dieser Zeit hat er der Schweiz 164. Chefchirurgen, Professoren, Primarien und Abteilungsleiter ausgebildet. Sie alle hat er mit der Art und Kunst seines Lehrens fasziniert. Zum einen Teil ist sie begründet in der Einmaligkeit seiner persönlichen Lehrbegabung, dem Erbstück aus einer bekannten altösterreichischen Lehrerfamilie. Mit dieser Begabung hat dieser Enkelschüler ein Vierteljahrhundert nach dem Tode Billroths dessen Vorstellung vom Lehren als einer magisch hervorbrechenden und andere magisch ergreifenden Urmacht ${ }^{42}$ in einer Weise verwirklicht, die B. Breitner schlechtweg als «Genie des Lehrens ${ }^{43}$ bezeichnen konnte. Zum andern aber gründet sich Clairmonts Zürcher Lehrerfolg auch auf eine große Lehrerfahrung und auf bestimmte Lehrusancen und ist insofern einer rationalen Analyse zugänglich.

Sehen wir uns einmal die Maßnahmen an, mit denen er der Zürcher Klinik neue, «bisher nicht ausgebeutete Lernmöglichkeiten» (A. Ritter) erschloß. A. Ritter ${ }^{44}$ weiß darüber in seiner Gedenkansprache folgendes zu berichten: «In der Viertelstunde vor Beginn der Hauptvorlesung wurden durch Oberärzte und Assistenten kleinchirurgische, meist poliklinische Fälle in Gruppen den Studenten demonstriert und erklärt, so daß ein jeder mit Eintritt ins Klinikum von Anfang an in engere Fühlung mit der "Praxis“ kam. Ebenso geschätzt waren ... die Aufgebote zur Teilnahme an zystoskopischen, rektoskopischen und anderen Untersuchungen ... Mit besonders großer Begeisterung widmeten sich die Praktikanten dem ,24 stündigen Dienst ${ }^{6}$. Er gab ihnen Gelegenheit, in der Klinik mitzuarbeiten, bei konservativer Frakturenbehandlung und bei Programmoperationen zu assistieren, bei Notfällen deren rasche Abklärung, die gezielte Indikationsstellung, die angemessenen, dringlichen Eingriffe und die postoperative Nachbehandlung aus der Nähe kennenzulernen.»

Alle von Ritter aufgeführten Maßnahmen haben das gemeinsam, daß sie darauf zielen, einen vom Theoretischen überwucherten und durch passive Teilnahme der Studenten bestimmten Unterrichtsbetrieb vom Praktischen und Demonstrativen her aufzulockern und durch aktive Beteiligung der Studenten attraktiver und effizienter zu gestalten. Damit wird in den Bemühungen Clairmonts um die Reformierung des Zürcher Chirurgen-Unter-

42 Vgl. Theodor BiLlRoth, Über Lehren und Lernen der medizinischen Wissenschaften an den Universitäten der deutschen Nation, Wien 1876, S. $359 \mathrm{f}$.

43 Burkhard Breitner, Das Genie des Lehrens, Basel/Innsbruck 1948.

${ }^{44}$ In der Anm. 40 zit. Publikation. 
richts genau jener Zug der Wiener Schule offenbar, den Sonderegger 55 Jahre zuvor an ihr so rühmte: ihre Orientierung auf die ärztliche Praxis hin. Fragen wir jetzt, was das Eigentümliche in der durch Clairmont repräsentierten Schweiz-Österreich-Beziehung ist, so werden wir nach dem Dargelegten sagen können: Mit Clairmont hat Österreich ein Musterexemplar seiner guten, alten, in die Zeiten van Swietens und de Haens zurückreichenden medizinischen Lehrkultur auf helvetischen Boden verpflanzt.

Im selben Jahre 1919, in dem Clairmont nach Zürich berufen wurde, folgte auch der ehemalige oberste Hygieniker der österreichischen Armee, Robert Doerr (1871-1952) ${ }^{45}$, einem Ruf auf die Lehrkanzel für Hygiene und Bakteriologie an die Universität Basel. Er gehörte jener Gruppe österreichischer Immunologen an, die wie Rudolf Kraus, Karl Landsteiner und Clemens von Pirquet sich seit Beginn des Jahrhunderts diesem jüngsten Wissenschaftszweig widmeten und mit ihren Entdeckungen weithin fachbegründend wirkten ${ }^{46}$. Es ist kein Zufall, daß von diesen vier Pionieren nach dem Zusammenbruch der Donaumonarchie drei, Doerr, Landsteiner und Kraus, das klein und arm gewordene Österreich verließen. Der große Ausverkauf der Wiener Medizinischen Schule hatte begonnen. Für die Situation, in der sich Männer wie Doerr nach dem Zusammenbruch befanden, ist eine Notiz sehr aufschlußreich, die wir in der Selbstbiographie des Wiener Internisten und ehemaligen Mitarbeiters Doerrs während des Ersten Weltkriegs, Herbert Elias ${ }^{47}$, lesen: «Mein Freund und früherer Vorgesetzter, Professor Robert Doerr, später Professor der Hygiene an der Universität Basel und Hygieniker der League of Nations, konnte sich infolge der Entwertung seiner Pension nicht mehr recht ernähren. Seine rasche Gewichtsabnahme mußte jedem auffallen ...»

Doerr war damals bereits ein international anerkannter Immunologe und Allergologe. Wie die andern genannten Pioniere kam er vom serologischen Laboratorium Richard Paltaufs im Rudolfspital, in das er bald nach seiner Promotion (1897) eingetreten war. 1905 war er bereits Chef des Bakteriologischen Laboratoriums der österreichischen Armee. Als deren oberster

45 Vgl. Fritz Reuter, In memoriam Robert Doerr, Wiener klin. Wschr. 69 (1952) 129 f.; J.Tomcsiк, Robert Doerr, Bull. Schweiz. med. Wiss. 8 (1952) $320 \mathrm{f}$.

${ }^{46}$ Dazu vgl. E.Lesky (zit. Anm.16) S. 574 ff.; dieselbe, Viennese Serological Research around 1900, im Druck.

47 Herbert Elias, Erinnerungen eines österreichischen Arztes, 1885-1938. Unveröffentlichtes Manuskript, Handschriftensammlung, Institut für Geschichte der Medizin, Wien, Nr. 1986. 
Hygieniker hat er sein epidemiologisches Meisterstück vollbracht und die Heimat vor der bereits an der Ostfront herrschenden Cholera bewahrt.

Diesen erfahrenen Seuchenhygieniker hat sich 1919 die Schweiz geholt und damit gleichzeitig einen führenden Allergologen gewonnen. Zweimal hat sich in den zwanziger Jahren die Möglichkeit ergeben, ihn nach Wien zurückzuholen, d.h. auf die freigewordene Lehrkanzel für Hygiene bzw. auf jene der experimentellen Pathologie zu berufen. $\mathrm{Da} \beta$ man dies jedoch nicht tat, bezeichnete Wagner-von Jauregg ${ }^{48}$ in seinen Lebenserinnerungen als eines der bedrohlichsten Zeichen der damaligen Entwicklung.

Ich habe Sie in unserem Medicorum commercium einen weiten Weg geführt, der von dem sanktgallischen Humanisten Vadian bis zum obersten Armeehygieniker Österreichs reichte. Es hat sich gezeigt, daß auf diesem Wege beide Länder sehr gut gefahren sind und sich auf den verschiedensten Gebieten ärztlicher Tätigkeit gegenseitig fördern und ergänzen konnten. $\mathrm{Ja}$, wir sind auf diesem Wege nicht wenigen Zeugnissen einer echten amicitia medica Austro-Helvetica begegnet. Es ist für mich eine tiefe Freude feststellen zu können, daß sich diese im Wiener Medizinhistorischen Institut so gut wie täglich in lebhaften Kontakten mit der Schweizer Forschung bekundet. Nehmen Sie es als kleines Zeichen dieser Verbundenheit mit Schweizervolk und Schweizerland, daß dieser Vortrag unweit der Stadt Vadians konzipiert und niedergeschrieben wurde.

${ }^{48}$ Vgl. J. WAGNER-JAUREGG, Lebenserinnerungen, herausgegeben und eingeleitet von L. Schönbauer und M. JANTSCH, Wien 1950, S. 84 f. 